\section{Quantum simulation of black-hole radiation}

of ultracold atoms (Fig. 1b). In this approach, part of the fluid travels at a speed that is equal to or greater than the propagation speed of sound waves in the medium. A sound wave that is produced inside this region must follow the fluid flow because it cannot propagate in the opposite direction to the strong current. The outer edge of this area therefore forms an analogue black-hole event horizon. More importantly, the evolution of sound waves in such a fluid can be made to mimic exactly the propagation of classical or quantum fields near a black-hole event horizon ${ }^{4-7}$. As a result, the fluid can be used as a quantum simulator for black-hole radiation, when it is operated using a high level of control and at sufficiently low temperatures ${ }^{8}$.

The work of de Nova and colleagues builds on several experimental investigations in which this approach was used to set up analogue black-hole event horizons ${ }^{9}$ to study Hawking radiation ${ }^{10}$. The authors use a state of matter called a Bose-Einstein condensate that consists of 8,000 rubidium- 87 atoms. They use one laser beam to confine the condensate and another to generate a downward potential step - a region in which the potential energy drops sharply. This step moves through the condensate at a constant speed, which is equivalent to the condensate travelling at a constant speed in the reference frame in which the step is stationary. The condensate that flows over the step is accelerated to supersonic speeds, thereby forming an analogue black-hole event horizon.

The authors show that pairs of sound waves are produced at this event horizon. One wave of the pair is emitted away from the supersonic region in the form of Hawking radiation and the other, which has a negative energy, is absorbed into this region (Fig. 1b). Hawking predicted that a black hole will emit radiation that can be described by a single Hawking temperature, dependent only on the mass of the black hole and not on the details of the gravitational field that lies outside the event horizon. The main novelty of de Nova and colleagues' work is a clever detection scheme that they use to extract the temperature of the emitted radiation. The authors' findings provide the first evidence of the Hawking temperature from a quantum simulator.

The energy spectra of the emitted radiation lack traces of the microscopic nature of the system $^{5}$, as well as of macroscopic grey-body corrections. The latter concern the reflection of the emitted radiation back towards the event horizon, owing to an effective potential-energy variation outside the horizon. Such backreflection is expected to occur for astrophysical black holes. In quantum simulators for blackhole radiation, these microscopic and macroscopic effects can be tuned ${ }^{11}$, and their absence demonstrates the high level of control that de Nova et al. exert over their experimental apparatus. The authors' set-up is promising, and could be used to investigate many other

Modelling black holes in the laboratory. a, An astrophysical black hole is characterized extremely warped region of space-time. In the 1970s, Stephen Hawking studied ${ }^{1}$ what happens when pair of particles is produced from the vacuum of space near a black hole's event horizon - the boundary beyond which nothing can escape. He found that one of the particles would be absorbed by the black hole, and the other would be emitted into space in the form of thermal radiation, which is now called Hawking radiation. $\mathbf{b}$, de Nova et al. ${ }^{3}$ report observations of an analogue black hole, which is based on a flowing fluid of ultracold atoms. One region of the fluid travels at a supersonic speed and a connected region travels at a subsonic speed; sizes of grey arrows indicate speed. The boundary between these regions provides an analogue black-hole event horizon. When a pair of sound waves is produced near this boundary, one of the waves is absorbed into the supersonic region, and the other is emitted away from the region in the form of Hawking radiation. 
interesting phenomena - for example, the quantum correlations that are expected to be exhibited by pairs of sound waves produced at the event horizon. -

Silke Weinfurtner is at the School of Mathematical Sciences, University of Nottingham, Nottingham NG7 2RD, UK. e-mail:silke.weinfurtner@nottingham.ac.uk

1. Hawking, S. W. Nature 248, 30-31 (1974).

2. Unruh, W. G. Phys. Rev. D 14, 870-892 (1976)

3. de Nova, J. R. M., Golubkov, K., Kolobov, V. I. \&
Steinhauer, J. Nature 569, 688-691 (2019).

4. Unruh, W. G. Phys. Rev. Lett. 46, 1351-1353 (1981).

5. Barceló, C., Liberati, S. \& Visser, M. Living Rev. Relativ. 14, 3 (2011)

6. Weinfurtner, S., Tedford, E. W., Penrice, M. C. J., Unruh, W. G. \& Lawrence, G. A. Phys. Rev. Lett. 106 021302 (2011).

7. Euvé, L.-P., Michel, F., Parentani, R., Philbin, T. G. \& Rousseaux, G. Phys. Rev. Lett. 117, 121301 (2016).

8. Garay, L. J., Anglin, J. R., Cirac, J. I. \& Zoller, P. Phys. Rev. Lett. 85, 4643-4547 (2000).

9. Lahav, O. et al. Phys. Rev. Lett. 105, 240401 (2010).

10.Steinhauer, J. Nature Phys. 12, 959-965 (2016).

11. Coutant, A. \& Weinfurtner, S. Phys. Rev. D 97 025006 (2018)

\section{Guarding the gate for mitochondrial entry}

\section{The protein-import systems of organelles can become clogged by proteins. A protein from one organelle, the endoplasmic reticulum, is found to also unclog such blockages in mitochondrial organelles. SEE ARTICLE P.679}

\section{SYLVIE CALLEGARI \& PETER REHLING}

$\mathrm{O}$ rganelles known as mitochondria are the energy-generating powerhouses of the cell. As a crucial part of the cellular machinery, disruption to their function could have serious consequences, and so mechanisms exist to combat mitochondrial dysfunction. On page 679, Mårtensson et al. ${ }^{1}$ report a pathway that tackles problems concerning the import of proteins into mitochondria, as they arise, to prevent mitochondrial and cellular dysfunction. This pathway uses part of the cellular repair kit that handles damaged proteins in another organelle - the endoplasmic reticulum (ER).

At least 1,000 proteins reside within the mitochondria of yeast, where they carry out functions that contribute to cellular energy production and metabolism ${ }^{2}$. Almost all of these proteins are made in the cytoplasm and are then transported into mitochondria in an unfolded precursor form. Mitochondria receive a heavy inbound flow of protein traffic, and the entry route that bears the brunt of this influx is a protein complex on the mitochondrial surface called the TOM complex. When the TOM complex becomes overloaded or is compromised due to mitochondrial damage, unfolded mitochondrial proteins can accumulate in the cytoplasm and cause cellular toxicity $^{3-6}$. Mechanisms exist to combat the stress to cells that results from this ${ }^{3-6}$, but how mitochondria handle protein-import failure as it arises was unclear.

The pore of a TOM complex will sometimes become clogged with a mitochondrial protein (Fig. 1). Protein misfolding is a common cellular event that can be exacerbated by stress or occur if a protein is in a mutant form ${ }^{7}$. A mitochondrial precursor protein that folds prematurely in the pore of the TOM complex could become stuck there. A blockage might also occur if the energy levels of the cell drop, leading to a slowing or stalling of protein import ${ }^{8}$. If such an obstruction is not removed, the accumulation of unfolded precursor proteins in the cytoplasm can trigger transcriptional changes that lead to the unleashing of a cellular stress response ${ }^{4}$. Such stress-response pathways ${ }^{3,4}$ include the UPRam pathway and the mitoCPR pathway, which prime the cytoplasmic waste-disposal system - a protein complex called the proteasome - to degrade the accumulated precursor proteins.

Mårtensson et al. investigated whether a system is in place to constantly monitor the TOM complex and to remove trapped precursor proteins, thereby preventing blockages from hampering protein import to an extent that causes cellular stress. Such a monitoring system is in place for an entry gate into the ER ${ }^{9}$. To identify possible factors that might clear away trapped precursor proteins, Mårtensson $e t$ al. purified the TOM complex from the mitochondria of yeast cells. One of the TOM-complex-associated proteins that they identified was Ubx2, which was a surprise. This is because Ubx2 is best known for its activity at the ER, where it functions in the routine clearance of misfolded proteins as part of an ER-specific quality-control pathway called ER-associated degradation (ERAD) ${ }^{10,11}$. Mårtensson and colleagues conducted biochemical experiments that showed that Ubx2 exists in two distinct cellular pools, one at the ER and another on the mitochondrial surface

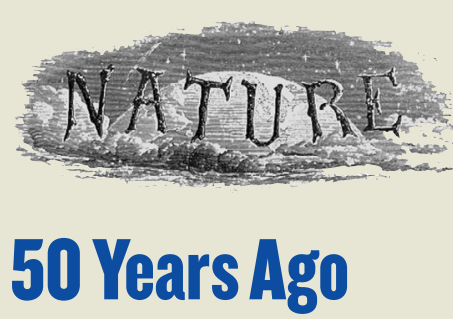

Apollo 10 has demonstrated the technical virtuosity of NASA and its works. It is hard now to remember that it is merely two years since an unlucky fire cost the lives of three astronauts at Cape Kennedy. Then it seemed as if the dream of getting to the Moon before the end of the sixties would be broken. Now, as the numbers tick by towards eleven, the meticulous care with which the whole enterprise has been conducted becomes increasingly apparent. The Apollo programme seems to be bursting with a host of technological sagas ... It is true that the United States is hoping that there will flow from this accumulation of expertise a further refinement of the skill ... which has made it the inventive place it is already ... But the more lasting achievement is the way in which the anticipation of technical problems has created a sense of mastery of machines ... The old cliché of the returning hero that it is the team which deserves the honours has become a truism. From Nature 31 May 1969

100 Years Ago

As the result of an executive order issued by President Wilson ... the temporary arrangement inaugurated two years previously has acquired permanence as the National Research Council of the United States. The history of this organisation is instructive in showing that in time of national stress the Governmental authorities appreciate the necessity for active co-operation from scientific bodies or individuals who have in peace conditions received but little recognition or support ... Thus it is that the ... direction of research work in the United States has become vested in a body of men whose ... work during the war ... has shown that they are competent to handle the great problems which go with peace and reconstruction. From Nature 29 May 1919 